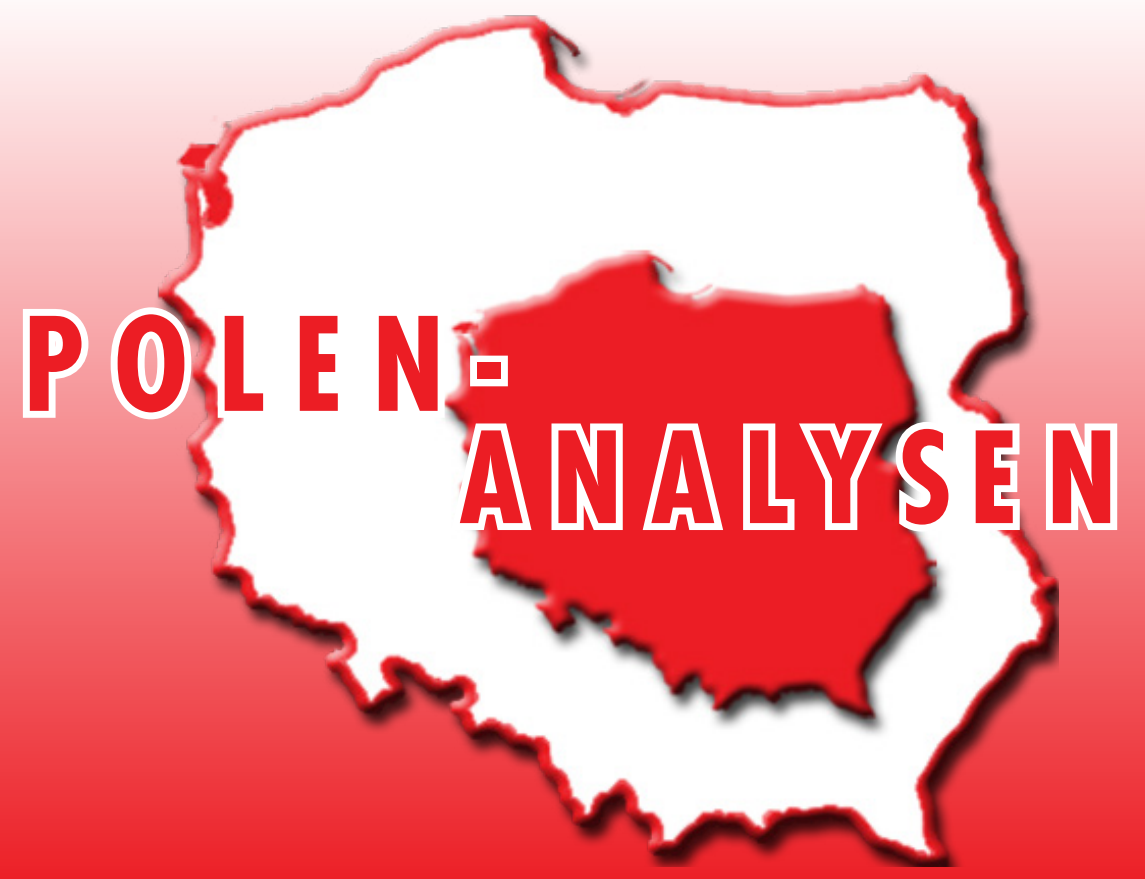

www.laender-analysen.de/polen

\title{
POLNISCHE ÜBERLEGUNGEN ZU EUROPA
}

\section{ANALYSE}

Polnische Überlegungen zu Europa

Andriy Korniychuk, Warschau

\section{CHRONIK}

20. September 2016 - 3. Oktober 2016

Herausgegeben mit finanzieller Unterstützung

der Stiftung für deutsch-polnische Zusammenarbeit

FUNDACJA WSPÓtPRACY

POLSKO-NIEMIECKIEJ

STIFTUNG

FÜR DEUTSCH-POLNISCHE

ZUSAMMENARBEIT

\section{DEUTSCHES POLEN INSTITUT}

- Forschungsstelle Osteuropa an der Universität Bremen
Deutsche Gesellschaft

DGO für Osteuropakunde e.V. 


\title{
Polnische Überlegungen zu Europa
}

\author{
Andriy Korniychuk, Warschau
}

\section{Zusammenfassung}

Das Referendum der Bürger Großbritanniens, das über den Austritt des Landes aus der Europäischen Union entschied, werten viele Experten und Entscheidungsträger als unaufschiebbare Aufforderung, Veränderungen im europäischen Integrationsprojekt vorzunehmen. Die größte Herausforderung für Europa besteht darin, eine Antwort auf die Fragen zu finden, wie das bis vor kurzem so erfolgreiche Projekt der europäischen Integration nun in eine multiple Krise geraten ist und wie es wieder herausfindet. Angesichts der enorm gewachsenen Herausforderungen ruft die Mehrheit der rechtskonservativen Politiker und der Europaskeptiker dazu auf, zu den Nationalstaaten, dem Europa der Vaterländer (Charles de Gaulle), zurückzukehren. Ein Teil der Experten ist überzeugt, dass ein Wendepunkt in der Integrationspolitik eingetreten sei, jedoch in dem rechtlich-institutionellen Rahmen der Europäischen Union in der gegenwärtigen Gestalt keine Antworten auf die neuen Herausforderungen gefunden werden können. In dem Beitrag soll die polnische Vision von der Zukunft Europas aktuell vor dem Hintergrund des Sondergipfels der Regierungschefs der Europäischen Union am 16. September 2016 in Bratislava und der Debatte über die Herausforderungen, vor denen die Mitgliedsstaaten stehen, dargelegt werden.

$\mathrm{D}$ ie Europäische Union wurde recht unglücklich die Geisel der Ängste der Bürger, die aus der Entwicklung der Globalisierung genährt werden: Die neuen Herausforderungen sind häufig supranational (beispielsweise die Migrationsprozesse, der Klimawandel), doch der gewöhnliche Bürger betrachtet die gemeinschaftliche Herangehensweise zunehmend als weiteres Problem und nicht als Lösung von Problemen. Die Stimme Polens in der gegenwärtigen Debatte über die Zukunft der Integration ist aus verschiedenen Gründen wichtig.

Erstens: In der polnischen politischen Elite gibt es viele Stimmen, die von der Notwendigkeit sprechen, die EU zu reformieren. Nachdem die Ergebnisse des Referendums in Großbritannien bekannt gegeben worden waren, erklärte Jarosław Kaczyński, der Vorsitzende der Regierungspartei Recht und Gerechtigkeit (Prawo i Sprawiedliwość-PiS), die Notwendigkeit, den europäischen Vertrag zu ändern. Seiner Meinung nach beendet das Konzept des "mehr EU« nicht die bestehenden Krisen. Aus Sicht der PiS sollte sich das neue Konzept eines vereinten Europa auf die Stärkung der Rolle der Nationalstaaten stützen und diesen Souveränität garantieren, unter anderem indem die Rolle der nationalen Institutionen, der Parlamente, in Entscheidungsprozessen auf übernationaler Ebene gestärkt wird. Dabei handelt es sich um keine vereinzelte Stimme in der europäischen Debatte über die Zukunft der Integration auf dem Kontinent.

Zweitens: Zu beachten ist außerdem ein Phänomen, das als "polnisches Paradox" bezeichnet werden kann. Es besteht darin, dass in einem Land, das von der Integration des Kontinents deutlich profitiert hat und profitiert, insbesondere im Zusammenhang mit Investitionen und dem Zugang zum gemeinsamen Markt, immer häufiger skeptische Stimmen in Bezug auf eine Vertiefung der Zusammenarbeit im Rahmen der EU zu vernehmen sind. Und dieses Paradox ist nicht nur in Polen anzutreffen. Versuchen wir also zu verstehen, ob das Konzept der gelockerten Zusammenarbeit in Europa, die sich vor allem auf die Wirtschaftsbeziehungen konzentriert und dabei den Mitgliedsstaaten eine größere politische Souveränität garantieren soll, die optimale Richtung für die Integration darstellt.

Bevor wir uns auf die polnische Perspektive konzentrieren, sollen die Prozesse und Phänomene betrachtet werden, die den Diskurs über die Zukunft der EU prägen. In der letzten Zeit wird immer dezidierter von der institutionellen Krise und dem Demokratiedefizit in der Gemeinschaft gesprochen. Auch ist von der Notwendigkeit, die Souveränität der Nationalstaaten zu verteidigen, zu hören. Die Stichhaltigkeit der Vorwürfe muss bewertet werden, zumal in den Debatten über die Zukunft der EU häufig Formulierungen verwendet werden, die vor allen Emotionen hervorrufen und davon abhalten sollen, sich mit den Herausforderungen selbst auseinanderzusetzen.

Des Weiteren: Die EU steht vor der Notwendigkeit, mit den Migrationsprozessen erfolgreich umzugehen. Die Herausforderungen im Zusammenhang mit der Migrationspolitik zwingen zur Auseinandersetzung mit den Themen Verteidigung und Sicherheit auf dem europäischen Kontinent. Sowohl die Bekämpfung extremistischer Bewegungen als auch die Notwendigkeit, die Außengrenzen zu schützen, wurden zu prioritären Aufgaben sowohl für die EU als auch für einzelne Mitgliedsstaaten. Zum ersten Mal seit Jahren kam es zu einer vorübergehenden regionalen Aufhebung des Schengenraums und zu Grenzkontrollen. Außerdem wird seit Monaten 
über ein mögliches Auseinanderbrechen der Eurozone gesprochen sowie über die Notwendigkeit, das Wirtschaftswachstum anzukurbeln und die Kohäsion der Mitgliedsstaaten zu fördern.

Es ist kein Zufall, dass die größten Herausforderungen für Europa in den Bereichen liegen, die zum Kompetenzbereich der Nationalstaaten gehören, so die Migration, der Grenzschutz usw. Das Problem ist hier nicht eine zu weit reichende Integration, sondern dass es sie in bestimmten Bereichen nicht in ausreichendem Maße gibt. Das bedeutet nicht, dass das europäische Integrationsmodell eine ideale Lösung wäre, die keiner weiteren Korrekturen bedarf. Der außergewöhnliche Charakter der regionalen Zusammenarbeit in Europa hat die dynamische Entwicklung der Gemeinschaft angetrieben. Im Grunde erinnert das Projekt der europäischen Integration seit Anbeginn an ein Schiff, an dem zahlreiche Umbaumaßnahmen durchgeführt werden, während es auf Fahrt ist. Die europäische Integration hat sich von Krise zu Krise entwickelt. Daraus ergibt sich die These, dass die neuen Herausforderungen eine weitere Chance für die EU sind, die Instrumente der Entscheidungsfindung weiterzuentwickeln, denn es gibt kein anderes Beispiel für eine so fortgeschrittene Integration globalen Ausmaßes.

\section{Alles beginnt mit Kommunikation}

Die Ausbreitung der Kultur der "sieben Sekunden" in den Medien (nicht nur im Internet, sondern zunehmend auch in den traditionellen Medien), die ihren Schwerpunkt auf kurze, bunte und nicht unbedingt inhaltliche Botschaften legt, scheint dem Populismus in Europa in die Hand zu spielen, für den Mittel wie die sozialen Medien ideal sind, um mit den Bürgern in Interaktion zu treten. Auf diese Herausforderung sucht die EU erst noch eine Antwort. Obgleich seit Jahren darüber gesprochen wird, fehlt der Union immer noch eine effektive Kommunikationsstrategie, die die Informationstechnologien und die sozialen Medien für die Beteiligung der Bürger an Entscheidungsprozessen nutzen würde. Allerdings widmet die EU Fragen der Kommunikation immer mehr Aufmerksamkeit, was auch der Sondergipfel in Bratislava am 16. September 2016 deutlich gemacht hat, auf dem dazu aufgerufen wurde, die Kommunikation zwischen den Mitgliedsländern, den Bürgern und den Institutionen zu verbessern.

\section{Naturalisation der EU}

Eine weitere ernstzunehmende Herausforderung ist für die EU die Naturalisation der europäischen Integration. Das »Europa der Vaterländer", um das sich ein Teil der Politiker bemüht, endete im vergangenen Jahrhundert mit zwei Weltkriegen und einer ganzen Weltregion, die im Chaos versank. Die EU stellte eine Lösung dar, die auf dem zerstörten Kontinent den lang erwarteten Frieden und Wohlstand bringen sollte. Daher war auch der Einspruch gegen den Ausbau der Integration sogar zwischen ehemaligen Feinden minimal.

Fast 60 Jahre nach Beginn der Integration haben wir es nun mit einer Generation von jungen Europäern zu tun, für die die Begriffe Krieg oder repressives Regime nur aus Erzählungen und audiovisuellem Material bekannt sind. Für die heutige Generation ist die Integration Europas zu einem selbstverständlichen Teil ihres Lebens geworden. Der Integrationsprozess wird von ihr ausschließlich unter dem Aspekt der sich daraus ergebenden Rechte und der Angebote gesehen, während die Pflichten, die sich aus der Gemeinschaft ergeben, beispielsweise der Wert der Solidarität, nicht berücksichtigt werden.

Die neuen Ungewissheiten und Angst vor dem Unbekannten bewegen auch viele junge Leute zur Rückkehr zu den Werten, die uns vertraut sind. So ist die Rückkehr zu den Werten der Nationalstaaten ein Verteidigungsmechanismus vor dem ungewissen Prozess einer vertieften Integration, die nicht selten mit der Globalisierung identifiziert wird. Immer populärer wird der Mythos, dass man innerhalb der Grenzen des Nationalstaates Lösungen anwenden kann, die alle Bedürfnisse der Bürger befriedigen.

Die EU wurde zur Geisel ihres eigenen Modells vom Wohlfahrtsstaat. Die institutionellen Reformen der Gemeinschaft, hinter denen ein zeitaufwändiger Prozess steht, der das Einverständnis aller Mitgliedsstaaten erfordert, halten offensichtlich nicht mit den sich verändernden Erwartungen der Bürger in einer sich dynamisch entwickelnden Welt mit. Kurzfristig muss die Gemeinschaft in den Augen der Gesellschaften Europas die operative Kompetenz wiedergewinnen und die eindeutige Botschaft aussenden, dass die Integration immer noch die bessere Lösung für die bestehenden Herausforderungen ist.

Die erste Lösung sollte eine Kommunikationspolitik sein, die die moderne Informationsvermittlung berücksichtigt. Zweitens sollte auf der Grundlage der Kommunikationsstrategie eine neue positive Narration der Europäischen Union erarbeitet werden. Die neue Narration ist eine Möglichkeit, die Richtung der erwünschten Veränderungen zu bestimmen. Der aktuelle Diskurs in der EU wurde zu einer Diskussion, deren Regeln von den Euroskeptikern und den Populisten vorgegeben wurden. Die Folge der Debatte, die bewusst bestimmte Emotionen wie Angst und Unsicherheit hervorrufen soll, ist eine schier endlose Polarisierung der Gesellschaften, wovon die populistischen Bewegungen profitieren. Die proeuropäischen Eliten wiederum konzentrieren sich fort- 
während darauf zu beschreiben, was sie nicht wollen wobei sie aber nicht eindeutig die Richtung benennen, in die sich Europa entwickeln sollte, um die Herausforderungen zu bewältigen.

\section{Was ist mit Polen?}

Solange die EU keine wirksame Lösung für eine Veränderung in der Kommunikation und für die Vertiefung des Integrationsprozesses findet, ist es im besten Interesse Polens, dass sich das Projekt der Zusammenarbeit in Europa auf zwei Grundsätze stützt auf die gemeinschaftsbezogene Herangehensweise an die Politiken und auf die institutionelle Kohäsion des Entscheidungsprozesses.

Eine fundamentale Infragestellung des Status quo ist der Vorschlag, den europäischen Vertrag zu ändern, der unter anderen vom PiS-Vorsitzenden Jarosław Kaczyński vorgebracht wurde. Allerdings ließe die Effektivität der avisierten Änderungen für Polen viel zu wünschen übrig, so lautet die Einschätzung eines Großteils der polnischen Experten. Sowohl in Brüssel als auch in vielen Mitgliedsstaaten stehen die politischen Eliten der Möglichkeit einer vollständigen Überprüfung des rechtlichinstitutionellen Rahmens der EU ablehnend gegenüber. Vertragsänderungen sind sogar in stabileren Zeiten ein zeitaufwändiger Prozess mit ungewissem Ausgang, der vonseiten der Mitgliedsstaaten viele Ressourcen erfordert. Solange der Austritt Großbritanniens aus der EU nicht formalisiert ist und die Union die aktuellen Herausforderungen bewältigen muss, scheint eine Verfassungsänderung wenig realistisch. Dies bestätigte der informelle Gipfel der EU-Regierungschefs in Bratislava, in dessen anschließend vorgestellter roadmap das Thema Vertragsänderungen keine wichtige Rolle spielte.

Die Investition politischen Kapitals in eine Änderung des Lissabon-Vertrages kann nach sich ziehen, dass insbesondere die Staaten des harten Kerns der EU (die zunehmend von einer Vertiefung der Zusammenarbeit sprechen) der Ansicht sind, dass die polnische Regierung nicht imstande ist, die Stimmung in der Gemeinschaft treffend zu diagnostizieren. Auch vonseiten der Staaten der Visegrád-Gruppe, deren Vorsitz zurzeit Polen innehat, ist keine wesentliche Unterstützung für so weitreichende Veränderungen in der Union festzustellen. Trotz Übereinstimmungen hinsichtlich der Notwendigkeit, manche Institutionen zu reformieren, ist in der Gruppe keine Unterstützung für eine »Öffnung der Verträge« zu sehen. Es ist also nicht damit zu rechnen, dass die polnische Außenpolitik Vertragsänderungen auf kürzere Sicht Priorität einräumt.

Eine weitere wichtige Forderung, die in Polen auftaucht, aber auch in einigen anderen Mitgliedsstaaten, ist die Stärkung der Rolle der nationalen Institutio- nen im Entscheidungsprozess der EU. Dies soll dem Demokratiedefizit in der Gemeinschaft entgegenwirken. Obgleich in der roadmap festgestellt wird, dass die EU ein Kommunikations- und nicht ein Demokratiedefizit hat, bedingt der dynamische Charakter der europäischen Integration, dass sich die Gemeinschaft in einer Phase ständiger Veränderungen befindet und ihr rechtlich-institutionelles System solcher Korrekturen bedarf, dass es den neuen Herausforderungen die Stirn bieten kann. Unbestritten ist der Prozess der demokratischen Legitimierung der EU noch weit von seinem Abschluss entfernt. Trotz gewisser Erfolge liegt vor Brüssel noch der lange Weg, ein demokratisches Modell auf supranationaler Ebene zu finden, das die Erwartungen der Experten und der Bürger der EU zufriedenstellt. Die Stärkung der nationalen Institutionen wäre dagegen eine wenig vorteilhafte Perspektive für Polen, und zwar aus diesen Gründen:

Aus der Befürchtung heraus, dass der Integrationsprozess sonst vonseiten der Länder der sogenannten alten Union dominiert wird, ist es logisch, die supranationalen Institutionen wie die Europäische Kommission oder das Europäische Parlament, die die Werte der Gemeinschaft schützen, zu stärken. Eine praktische Widerspiegelung dieser Werte kann beispielsweise die Verteilung der Mittel der EU-Fonds werden. Aus wirtschaftlicher Sicht sollte demnach Polen als Land, das vom Zugang zum Binnenmarkt und von der Kohäsionspolitik stark profitiert, daran interessiert sein, die relativ starke Position der supranationalen Organe aufrechtzuerhalten.

Die institutionellen Reformen sind mit Blick auf ein auch zukünftig erfolgreiches Integrationsprojekt langfristig wichtig. In mittel- und kurzfristiger Perspektive sollte allerdings für Polen das grundlegende Ziel sein, die Methode, in der EU gemeinschaftlich Politik zu betreiben, aufrechtzuerhalten. Die größte Herausforderung für das Land verbirgt sich hinter dem Phänomen des »Europa der unterschiedlichen Geschwindigkeiten«. Der Schengenraum und die Eurozone sind wahrscheinlich die gelungensten Beispiele für die Zusammenarbeit bestimmter Gruppen von Staaten in einem bestimmten Bereich. In Übereinstimmung mit dem Lissaboner Vertrag wurde in der EU der Mechanismus der verstärkten Zusammenarbeit eingeführt; wenn er in einem konkreten Fall von mindestens neun Staaten in Anspruch genommen wird, ist er offen für die übrigen Mitgliedsländer. Angesichts neuer Bedrohungen wird das »Europa der unterschiedlichen Geschwindigkeiten« immer häufiger als Modell diskutiert, das am besten den bestehenden und potentiellen Herausforderungen begegnet. Wird der Mechanismus dauerhaft eingesetzt, um die Politik in der EU zu steuern, kann es in der Gemeinschaft zu einer permanenten Teilung in verschiedene Integrationskreise 
kommen. Im für Polen ungünstigsten Fall entwickelt jede Gruppe in den Kreisen verstärkter Zusammenarbeit gesonderte Institutionen und rechtliche Lösungen.

Die Reduzierung der Bedeutung des gemeinschaftlichen Ansatzes als grundlegendes Prinzip der EU-Politik könnte also dazu führen, dass Polen in die B-Liga der Europäischen Union absteigt. Die Furcht, dass von den gemeinschaftlichen Lösungen Abstand genommen werden könnte, wird von Forderungen nach intensivierter Zusammenarbeit in der Eurozone sowie von den beunruhigenden Signalen im Zusammenhang mit den Austrittsverhandlungen Großbritanniens genährt (insbesondere das vorstellbare Zugeständnis an Großbritannien, die Personenfreizügigkeit beschränken zu dürfen bei gleichzeitigem Zugang zum Binnenmarkt). Abgesehen davon, dass für Polen die Zusammenarbeit in der NATO eine Sicherheitsgarantie darstellt, ist das gemeinschaftliche Auftreten im Rahmen der EU-Strukturen ebenfalls ein wirksames Instrument, der militärischen Aggression der Russischen Föderation entgegenzuwirken - hier wären beispielsweise die gelungenen Sanktionen der EU gegenüber Russland zu nennen. Konkret stellen sich die Herausforderungen für Polen in den dargestellten Bereichen folgendermaßen dar:

Aus der Perspektive eines Landes, das nicht Mitglied der Eurozone ist, ist die weitere Vertiefung der Zusammenarbeit im Rahmen unterschiedlicher Integrationskreise ohne aktive Beteiligung der draußen vor bleibenden Mitgliedsländer das am wenigsten vorteilhafte Szenario. Polen hat per Vertrag die Verpflichtung, der Eurozone beizutreten, was bisher nicht umgesetzt wurde, weil vor allem der politische Wille fehlte, die formale Entscheidung zu treffen. Obwohl in Polen das Verhältnis zu einer gemeinsamen Währung uneindeutig ist, stimmt der Kreis der Experten vor allem darin überein, dass das Szenario eines Zusammenbruchs der Eurozone wenig realistisch ist. Die Gestalt des gemeinsamen Marktes wird vielmehr von den Entscheidungen der Länder der Eurozone abhängen (ein Signal ist hier die Entsenderichtlinie für Arbeitnehmer). Selbst wenn das schlechteste Szenario, der Zusammenbruch der EU, vorgestellt wird, fällt auf, dass das Projekt einer neu zu konzipierenden Integration häufig im Zusammenhang mit einer gemeinsamen Geldpolitik besprochen wird.

Die höchste politisch-wirtschaftliche Priorität hat für Polen die Garantie der Inklusivität der Eurozone. Darunter ist nicht nur die Offenheit der verstärkten Zusammenarbeit gegenüber neuen Mitgliedern zu verstehen, sondern auch die Möglichkeit für die Staaten außerhalb der Eurozone (gegebenenfalls mit beschränktem Stimmrecht), an den Diskussionen über die Zukunft des gemeinsamen Marktes und der Geldpolitik teilzunehmen. Nach Meinung polnischer Experten ist es not- wendig, auf bereits bestehende institutionelle Lösungen zurückzugreifen, anstatt neue im Kontext der Bankenkrise in Italien anzuwenden. Auch die gemeinsame Energiepolitik ist aus der Sicht Polens eines der wichtigsten Ziele der EU in nächster Zukunft.

Die Vision Polens von Europa beinhaltet - unabhängig von der politischen Spaltung des Landes - die Unantastbarkeit der grundlegenden Freiheiten des Binnenmarktes. Die Verhandlungen über den Austritt Großbritanniens aus der EU sind ein Test für die Integrität der Gemeinschaft in Bezug auf die Achtung der Werte, für die das Integrationsprojekt steht. Großbritannien hat sich aus polnischer Perspektive sehr rasch von einem guten Freund zu einem ungeliebten Gast entwickelt. Bis zur Durchführung des Referendums über den »Brexit« kämpfte die britische Regierung sehr um die Rechte der Länder außerhalb der Eurozone, was sich bis zu einem gewissen Grad mit den Interessen Polens deckte. Aktuell stellen die Verhandlungen des "Brexit" eine ernste Bedrohung der Personenfreizügigkeit dar, zumal im öffentlichen Diskurs wiederholt Informationen verbreitet wurden, dass Großbritannien eventuell zugestanden werden würde, diese prinzipielle Freiheit im Rahmen der neuen kontinentalen Partnerschaft zu beschränken. Die aktive Beteiligung im Verhandlungsprozess mit dem Ziel, die Standhaftigkeit der EU bezüglich der vier Grundfreiheiten (freier Warenverkehr, Personenfreizügigkeit, Dienstleistungsfreiheit, freier Kapital- und Zahlungsverkehr) zu sichern, sollte eines der Hauptziele der polnischen politischen Eliten sein.

Auch mit Blick auf die in letzter Zeit heiß diskutierte Migrations- und Verteidigungspolitik ist festzustellen, dass aus polnischer Perspektive das gemeinsame Vorgehen die vorteilhafteste Lösung ist. Polen wird in Expertenkreisen als ein kulturell homogenes und vor allem als ein Auswanderungsland bezeichnet; der Ausländeranteil ist seit Jahren relativ gering. Die größte Gruppe von Ausländern stellen die kulturell nahestehenden Ukrainer. Bis zu den jüngsten Ereignissen in Europa spielte die Migrationspolitik keine größere Rolle im öffentlichen Diskurs des Landes. Die mangelnde Erfahrung mit kultureller Heterogenität und Immigration sowie die Instrumentalisierung der Ängste durch die Politik führten zu einer starken Polarisierung der Gesellschaft in der Frage der Aufnahme von Flüchtlingen. Der Einspruch der Regierung gegen eine Quotenregelung zur Verteilung der Flüchtlinge in der EU war ein Beitrag zu der allgemeinen Konfusion über die Zukunft der gemeinsamen Migrationspolitik.

Unter polnischen Experten und einem Teil der Politiker ist Unterstützung für eine gemeinsame Asylpolitik festzustellen, auch weil das Land seinen internationalen Verpflichtungen (Genfer Konvention) nachkommen muss. Die Einführung eines Quotensystems und von 
Sanktionen bei Nichtbeachtung werden in Polen vor allem als vorläufiges Instrument wahrgenommen, um der neuen Herausforderungen Herr zu werden. Interessant ist, dass Quotensysteme in der EU keineswegs neu sind, aber in einem so sensiblen Politikbereich wie der Migration wurde es Teil einer heftigen Debatte in ganz Europa. Aus polnischer Perspektive ist die Einrichtung eines Hilfsfonds für Personen, die sich um internationalen Schutz bemühen, eine viel wünschenswertere Lösung. Allerdings wäre bis zum Zeitpunkt der Verständigung über andere Lösungen wichtig, Solidarität mit den anderen Staaten zu zeigen. Die immer noch instabile Lage in der Ukraine fordert dazu auf, dass Polen mehrere Schritte vorausschauend reflektieren sollte. So kann sich die fehlende Unterstützung bei der Aufnahme auch nur einer kleinen Gruppe von Flüchtlingen für die Zukunft als politisch kostspielig erweisen.

Die Unterstützung für die Stärkung der Koordinationsrolle von FRONTEX ist ein weiterer Aspekt der Reformen der Union, den Polen nach Meinung von Experten unterstützen sollte. Der gemeinsame Grenzschutz ist eine Lösung, der auf keine eindeutige Reaktion der Mitgliedsstaaten stieß; jedoch wird die Intensivierung der institutionellen Zusammenarbeit und des Informationsaustausches bereits kurzfristig viel stärker messbaren Nutzen beim Schutz der Grenzen bringen. Der Gipfel von Bratislava bestätigt die Richtigkeit der dementsprechenden Vorschläge.

In der Frage der Verteidigung zählt Polen vor allem auf die Zusammenarbeit im Rahmen der NATO, was auf dem NATO-Gipfel in Warschau im Juli 2016 bestätigt wurde. Dessen ungeachtet wird die gemeinsame Verteidigungspolitik von der Regierung zunehmend als das Element wahrgenommen, das die EU-Staaten verbinden kann; insbesondere die Euroskeptiker sprechen sich nicht selten für die Notwendigkeit einer gemeinsamen Verteidigung Europas gegenüber militärischen Bedrohungen aus. Für Polen ist eines der Hauptziele, auf internationaler Ebene effektive Lösungen angesichts der Präsenz der Russischen Föderation auf ukrainischem Staatsgebiet und der Missachtung des Völkerrechts auszuarbeiten. Die Sanktionen der EU gegenüber Russland stießen auf ein positives Echo in Polen, und viele Experten verbinden den Erfolg die- ses Unternehmens mit einer gemeinschaftlichen Herangehensweise an neue Herausforderungen. In der Wahrnehmung polnischer Experten sollte die Verteidigung in einen größeren Kontext gestellt werden, der auch Antipropagandamaßnahmen mit einbezieht, die aktuell gegen die Russische Föderation eingesetzt werden. Initiativen wie East StratCom, deren Ziel es ist, russischen Desinformationskampagnen in Osteuropa entgegenzuwirken, sollten verstärkt werden, um Gefahren auch auf dem Territorium der Europäischen Union wirksam zu bekämpfen.

\section{Schlussbemerkung}

Der technologische Umbruch hat zur Folge, dass die Information zu dem am schnellsten konsumierten Produkt wurde. Anstatt das alte Telefon zu reparieren, kaufen wir ein neues, anstatt einer tiefer gehenden Analyse eines Problems auf der Grundlage von Daten und Literatur wählen wir tweets und Einträge auf Facebook. Diese kurze Illustration lässt sich auch auf ein so komplexes Gebilde wie die Europäische Union übertragen. Stimmen, die EU »in den Müll zu werfen« anstatt sich Gedanken über ihre Reparatur zu machen, treten immer häufiger im öffentlichen Diskurs auf. Die technologische Entwicklung, das neue Ausmaß der zwischenmenschlichen Kommunikation und die Vertiefung des Integrationsprozesses haben den Charakter der Herausforderungen, die vor der Europäischen Union stehen, wesentlich verändert

In der Medizin gilt die Krise als Moment des Umbruchs, der darüber entscheidet, ob der Patient seine Gesundheit wiedererlangt oder stirbt. Die dynamische Entwicklung des Integrationsprojekts in Europa, das im Laufe der Jahre auf große Herausforderungen stieß, erlaubt die Zuversicht, dass die EU immun vor gefährlichen Viren ist. Bevor wir eine Konterrevolution in Europa beginnen (zu der unter anderen Viktor Orbán aufruft), geben wir uns Zeit, das zu verbessern, was wir bereits haben. Aus der Perspektive Polens wäre die ideale Lösung für die neuen Herausforderungen die Förderung der gemeinschaftlichen Herangehensweise bei gleichzeitiger Garantierung der rechtlichinstitutionellen Kohäsion der EU.

Übersetzung aus dem Polnischen: Silke Plate

Die dargestellten Reflexionen wurden von den Expertendiskussionen im Rahmen der paneuropäischen Initiative »New Pact for Europe« inspiriert, deren Ziel die Erarbeitung eines "Vorschlagspakets« zu Veränderungen für das Funktionieren der EU und ihrer Politik ist.

\section{Über den Autor}

Andriy Korniychuk ist wissenschaftlicher Mitarbeiter des Instituts für Öffentliche Angelegenheiten (Instytut Spraw Publicznych - ISP), Warschau, und Mitglied der Gruppe für Europäische Studien des Instituts für Philosophie und Soziologie der Polnischen Akademie der Wissenschaften (IFIS PAN). Seine Forschungsinteressen sind die Legitimation des Systems des supranationalen Verwaltungshandelns in der EU, die Demokratie auf supranationaler Ebene und die Rolle der Bürgergesellschaft in den Modellen repräsentativer und deliberativer Demokratie. 


\section{September 2016 - 3. Oktober 2016}

\begin{tabular}{|c|c|}
\hline 20.09 .2016 & $\begin{array}{l}\text { Nachdem die Europäische Kommission am Vortag die Rechtmäßigkeit des Gesetzes zur Besteuerung des Ein- } \\
\text { zelhandels (seit } 1 \text {. September) in Frage gestellt hat, kündigt Ministerpräsidentin Beata Szydło an, gegen die Ent- } \\
\text { scheidung der Europäischen Kommission gerichtlich vorzugehen, sollte sich herausstellen, dass es dieser darum } \\
\text { ginge, das Vorgehen des polnischen Staates anders zu bewerten als das anderer Staaten. Die Europäische Kom- } \\
\text { mission prüft zurzeit, ob das Gesetz kleine Geschäfte bevorzugt. Bis zur Klärung hat Finanzminister Paweł } \\
\text { Szałamacha das Gesetz aufgehoben. }\end{array}$ \\
\hline 21.09 .2016 & $\begin{array}{l}\text { Jacek Protasiewicz, ehemaliger Abgeordneter der Bürgerplattform (Platforma Obywatelska-PO), teilt die Grün- } \\
\text { dung des Abgeordentenkreises Europäische Demokraten (Europejscy Demokraci) mit. Der Name umfasse zwei } \\
\text { Bereiche, die aktuell von der Regierungspartei Recht und Gerechtigkeit (Prawo i Sprawiedliwość - PiS) bedroht } \\
\text { würden. Weitere Gründungsmitglieder sind Stanisław Huskowski und Michał Kamiński. Alle drei waren im } \\
\text { Juli aus der PO wegen parteischädigenden Verhaltens ausgeschlossen worden. Der PO-Führung werfen sie vor, } \\
\text { sich nur mit sich selbst zu beschäftigen, anstatt PiS entgegenzutreten. }\end{array}$ \\
\hline 22.09 .2016 & $\begin{array}{l}\text { Die Tageszeitung »Gazeta Wyborzca« veröffentlicht eine Erklärung von } 19 \text { ehemaligen oppositionellen Aktivis- } \\
\text { ten des Komitees zur Verteidigung der Arbeiter (Komitet Obrony Robotników - KOR), in der diese ihre Teil- } \\
\text { nahme an der von Präsident Andrzej Duda ausgerichteten Feier zum 40. Jahrestag von KOR absagen. Duda } \\
\text { breche die polnische Verfassung und unterstütze die Regierung dabei, das Verfassungstribunal zu lähmen, heißt } \\
\text { es in der Erklärung. Dies widerspreche den Idealen, für die sie in der Volksrepublik Polen gekämpft hätten. }\end{array}$ \\
\hline 23.09 .2016 & $\begin{array}{l}\text { Der Sejm debattiert über zwei Bürgergesetzesinitiativen zum Abtreibungsrecht. Das Gesetzesprojekt »Stop abor- } \\
\text { cji«, das ein vollständiges Abtreibungsverbot vorsieht, wird mit } 267 \text { Stimmen (154 Gegenstimmen, elf Enthal- } \\
\text { tungen) zur weiteren Bearbeitung an den Ausschuss für Gerechtigkeit und Menschenrechte weitergeleitet. Das } \\
\text { Projekt »Ratujmy kobiety«, das die bedingungslose Möglichkeit einer Abtreibung bis zur 12. Schwangerschafts- } \\
\text { woche vorsieht sowie darüber hinaus bei Gefahr für Leben und Gesundheit der Mutter, bei schwerer Behin- } \\
\text { derung oder unheilbarere Krankheit des Kindes oder nach einer Vergewaltigung, wird mit } 230 \text { Stimmen (173 } \\
\text { Gegenstimmen, } 15 \text { Enthaltungen) nach der ersten Lesung abgelehnt. }\end{array}$ \\
\hline 24.09 .2016 & $\begin{array}{l}\text { In Warschau findet auf Initiative des Komitees zur Verteidigung der Demokratie (Komitet Obrony Demokra- } \\
\text { cji - KOD) erneut eine Großdemonstration gegen die Politik der Regierungspartei Recht und Gerechtigkeit } \\
\text { (Prawo i Sprawiedliwość - PiS) statt. Die Redner gehen vor allem auf die Krise um das Verfassungstribunal } \\
\text { und die Bildungspolitik der Regierung ein. Nach Schätzungen der Polizei nehmen 12.000, nach Angaben der } \\
\text { Warschauer Stadtregierung } 30.000 \text { Personen teil. }\end{array}$ \\
\hline 26.09 .2016 & $\begin{array}{l}\text { In Warschau treffen sich Vertreter der Ministerpräsidenten sowie der Außen- und Finanzministerien der EU- } \\
\text { Länder, die nicht der Eurozone angehören. Thematisiert wird das Kräfteverhältnis zwischen den Ländern mit } \\
\text { und ohne gemeinsame Währung, das sich infolge des »Brexit« verändern wird. Der Minister für Angelegen- } \\
\text { heiten der Europäischen Union, Konrad Szymański, sagt, Polen sei an einer Gesundung und an notwendigen } \\
\text { Reformen für die Eurozone interessiert, es müsse aber die Integrität des gemeinsamen Marktes gewahrt bleiben. }\end{array}$ \\
\hline 27.09 .2016 & $\begin{array}{l}\text { Der Pressesprecher der Bürgerplattform (Platforma Obywatelska - PO), Jan Grabiec, zeigt sich beunruhigt } \\
\text { über die Pläne von Verteidigungsminister Antoni Macierewicz, die Militärische Territoriale Verteidigung (Woj- } \\
\text { skowa Obrona Terytorialna - WOT) als fünfte Teilstreitkraft (neben den Boden- und den Luftstreitkräften, } \\
\text { der Marine und den Spezialkräften) zu gründen. Sollte sie, wie angekündigt, dem Verteidigungsminister und } \\
\text { nicht wie üblich dem Generalstab unterstehen, handele es sich um die private Armee von Macierewicz. Die PO } \\
\text { fordert die Entlassung des Verteidigungsministers. }\end{array}$ \\
\hline 28.09 .2016 & $\begin{array}{l}\text { Ministerpräsidentin Beata Szydło gibt bekannt, dass Mateusz Morawiecki als Finanzminister berufen wird. } \\
\text { Außerdem bleibt er Entwicklungsminister. Die Ministerien werden nicht zusammengefasst. Morawiecki wird des } \\
\text { Weiteren das neu berufene Ökonomische Komitee beim Ministerrat koordinieren. Für den bisherigen Finanz- } \\
\text { minister Paweł Szałamacha kündigt Szydło eine verantwortungsvolle Position an. }\end{array}$ \\
\hline 29.09 .2016 & $\begin{array}{l}\text { Bei seinem zweitägigen Besuch in den USA trifft sich Verteidigungsminister Antoni Macierewicz mit Senator } \\
\text { John McCain, dem Vorsitzenden des Ausschusses für Streitkräfte beim Senat. Thematisiert werden die gegen- } \\
\text { wärtige Lage und mögliche Entwicklungen in der Ukraine, die Stationierung von NATO-Truppen in Polen und } \\
\text { den baltischen Staaten, der Krieg in Syrien und ein mögliche neue Flüchtlingswelle nach Europa. }\end{array}$ \\
\hline 30.09 .2016 & $\begin{array}{l}\text { In einem Interview für die Tageszeitung "Dziennik« sagt Ministerpräsidentin Beata Szydło, dass es bei der Dis- } \\
\text { kussion über die Reformierung der EU keine Tabus geben dürfe, auch die Änderung des EU-Vertrages müsse } \\
\text { angesprochen werden können. Die nationalen Parlamente sollten gestärkt werden und die Europäische Kom- } \\
\text { mission sollte zu ihrer eigentlichen Funktion, eine verwaltende Institution zu sein, zurückkehren und nicht wie } \\
\text { zurzeit Politik betreiben. }\end{array}$ \\
\hline
\end{tabular}




\begin{tabular}{|l|l|}
\hline 02.10.2106 & $\begin{array}{l}\text { Auf dem Parteitag der Bürgerplattform (Platforma Obywatelska - PO) in Danzig (Gdańsk) kündigt der Par- } \\
\text { teivorsitzende Grzegorz Schetyna an, bei einem Machtwechsel das Institut des Nationalen Gedenkens (Insty- } \\
\text { tut Pamięci Narodowej - IPN) und das Zentrale Antikorruptionsbüro (Centralne Biuro Antykorupcyjne - } \\
\text { CBA) aufzulösen. Beide Institutionen würden gegenwärtig politisch instrumentalisiert und die Spaltung der } \\
\text { Gesellschaft befördern. }\end{array}$ \\
\hline 03.10.2016 & $\begin{array}{l}\text { Nach Meldungen des TV-Senders »TVP Parlament« sieht der Gesetzentwurf des Verteidigungsministeriums } \\
\text { über die Militärische Territoriale Verteidigung (Wojskowa Obrona Terytorialna - WOT) vor, die Teilstreit- } \\
\text { kraft mit ca. 35.000 Soldaten auszustatten. Die Kosten der Aufstellung der WOT sollen sich im Jahr } 2016 \text { auf } \\
\text { 394,4 Mio. Zloty belaufen. }\end{array}$ \\
\hline
\end{tabular}

Sie können die gesamte Chronik seit 2007 auch auf <http://www.laender-analysen.de/polen/> unter dem Link »Chronik«lesen. 
Die Polen-Analysen erscheinen zweimal monatlich als E-Mail-Dienst. Sie werden gemeinsam vom Deutschen PolenInstitut Darmstadt, der Bremer Forschungsstelle Osteuropa und der Deutschen Gesellschaft für Osteuropakunde herausgegeben.

Ein Archiv der Polen-Analysen finden Sie im Internet unter <www.laender-analysen.de/polen>

Kostenloses Abonnement unter <http://www.deutsches-polen-institut.de/Newsletter/subscribe.php >

Diese Analysen finden Sie online als Lizenzausgabe auf

$<$ bpb.de>

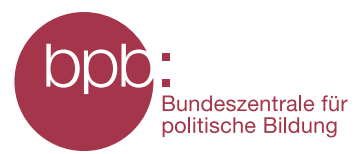

Deutsches Polen-Institut Darmstadt (<www.deutsches-polen-institut.de $>$ )

Das seit 1980 tätige Deutsche Polen-Institut Darmstadt (DPI) ist ein Forschungs-, Informations- und Veranstaltungszentrum für polnische Kultur, Geschichte, Politik, Gesellschaft und die deutsch-polnischen Beziehungen, die sich im Kontext der europäischen Integration entwickeln. Institutionelle Träger des DPI sind das Land Hessen, die Kultusminister der Länder, das Auswärtige Amt und die Wissenschaftsstadt Darmstadt. Einen wesentlichen Beitrag zur Verwirklichung der Institutsziele leisten private Stiftungen. Ziel der Vermittlertätigkeit des DPI ist es, „die zu interessieren, auf die es politisch, wirtschaftlich, gesellschaftlich und kulturell im deutsch-polnischen Verhältnis ankommt" (Leitlinien 1997). Es geht um die Entscheider und Multiplikatoren in Politik, Kultur, Bildung, Verwaltung, Medien und Wirtschaft. Das DPI versteht sich in Kooperation mit den Orten wissenschaftlicher Polen-Kompetenz an deutschen Hochschulen und Forschungsinstituten als verbindendes und vernetzendes Zentrum. Mit der über 68.000 Bände zählenden multidisziplinären Fachbibliothek für Polen, die eine einzigartige Sammlung polnischer Belletristik in der Originalsprache und in deutscher Übersetzung umfasst, ist das DPI ein geschätzter Ort der Recherche und des wissenschaftlichen Arbeitens.

Forschungsstelle Osteuropa an der Universität Bremen (<www.forschungsstelle.uni-bremen.de $>$ )

1982 gegründet, widmet sich die Forschungsstelle Osteuropa an der Universität Bremen der interdisziplinären Analyse der Länder Ost- und Ostmitteleuropas in Zeitgeschichte und Gegenwart. Der Forschungsschwerpunkt liegt dabei auf der Rolle von »Dissens und Konsens«, von Opposition und Zivilgesellschaft in ihrem historischen, politischen, gesellschaftlichen und kulturellen Kontext. Die Forschungsstelle besitzt in ihrem Archiv eine einzigartige Sammlung alternativer Kulturgüter und unabhängiger Texte aus den ehemaligen sozialistischen Ländern. Darunter befindet sich auch eine umfangreiche Sammlung des "Zweiten Umlaufs», die das Schrifttum und Dokumente unabhängiger Initiativen und gesellschaftlicher Gruppen in Polen aus der Zeit von 1976 bis zum Umbruch umfasst. Hinzu kommt eine umfangreiche Bibliothek mit wissenschaftlicher Literatur. Mit Archiv, Bibliothek und zwei wissenschaftlichen Abteilungen ist die Forschungsstelle auch eine Anlaufstelle sowohl für Gastwissenschaftler als auch für die interessierte Öffentlichkeit.

Eine der Hauptaufgaben der Forschungsstelle ist die Information der interessierten Öffentlichkeit. Dazu gehören unter anderem regelmäßige E-Mail-Informationsdienste für Politik, Wirtschaft, Zivilgesellschaft und Medien.

Das Institut für Öffentliche Angelegenheiten (Instytut Spraw Publicznych - ISP) in Warschau ist einer der führenden Think Tanks in Polen und seit 1995 als unabhängiges Forschungszentrum zu grundlegenden Fragen des öffentlichen Lebens tätig. Das ISP kooperiert eng mit zahlreichen Experten und Forschern wissenschaftlicher Einrichtungen im In- und Ausland. $<$ www.isp.org.pl>

Herausgegeben mit finanzieller Unterstützung der Stiftung für deutsch-polnische Zusammenarbeit

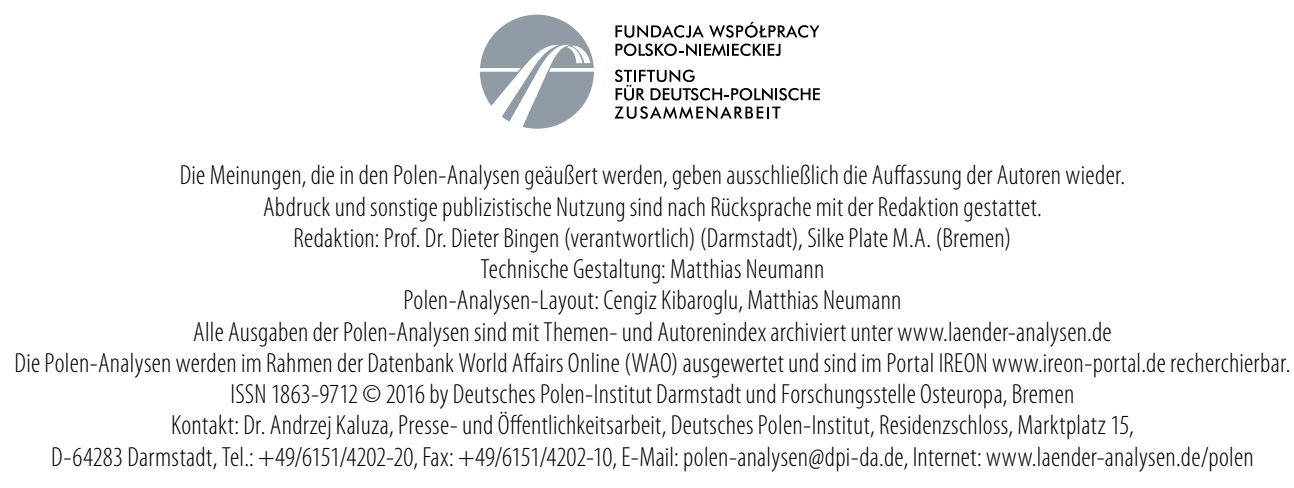

